\title{
GLAD!
}

Revue sur le langage, le genre, les sexualités

11 | 2021

Archives, genre, sexualités, discours

\section{Tordre les archives (queering archives) : oui, mais dans quel sens?}

Queering the Archives: in which Direction?

\section{Ruby Faure}

\section{(2) OpenEdition}

1 Journals

Édition électronique

URL : https://journals.openedition.org/glad/3255

DOI : $10.4000 /$ glad.3255

ISSN : 2551-0819

Éditeur

Association GSL

\section{Référence électronique}

Ruby Faure, «Tordre les archives (queering archives) : oui, mais dans quel sens ? », GLAD! [En ligne], 11। 2021, mis en ligne le 20 décembre 2021, consulté le 29 janvier 2022. URL : http:// journals.openedition.org/glad/3255; DOI : https://doi.org/10.4000/glad.3255

Ce document a été généré automatiquement le 29 janvier 2022.

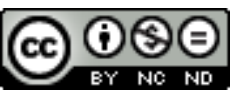

La revue GLAD! est mise à disposition selon les termes de la Licence Creative Commons Attribution Pas d'Utilisation Commerciale - Pas de Modification 4.0 International. 


\title{
Tordre les archives (queering archives) : oui, mais dans quel sens?
}

Queering the Archives: in which Direction?

\author{
Ruby Faure
}

1 Les batailles menées actuellement en France par le collectif Archives LGBTQI+ contribuent à déplacer ma relation aux archives de la sexologie dans l'Empire français à la fin $\mathrm{du} \mathrm{XIX}^{\mathrm{e}}$ siècle, autour desquelles je mène mes recherches de doctorat. Dans ces luttes épistémiques et politiques actuelles, l'explicitation de diverses formes de violences archivales (Zimmerman 2018) s'articule avec la volonté de penser des formes de re-présence capables de résister à l'effacement straight, institutionnel et étatique des histoires queer, mais aussi à l'intégration de ces histoires dans un récit étatique homonormatif blanchi, nationaliste et respectable (Bachetta 2018). Cette défense de nos archives queer fait pour moi écho avec le projet de «tordre les archives » (queering archives), selon le titre d'une double livraison récente de la Radical History Review ${ }^{1}$, qui a largement inspiré les questionnements épistémologiques, politiques et affectifs qui constituent la matière de cet article.

2 Je souhaite mener dans ce texte quelques allers-retours entre réflexions méthodologiques et lectures de matériaux historiques, en prêtant attention à la manière dont mes émotions et mes positions sociales et politiques participent à tordre (queer) ma relation au passé. Je me situe résolument dans l'héritage des épistémologies des savoirs situés, en considérant le rôle que joue ma propre subjectivité - produite par des rapports de pouvoir structurels - au sein du processus de ma recherche. Je comprends ici l'archive non comme un donné objectif ou neutre mais comme un artefact (Nestle 2015), une production historique façonnée par un ensemble de contraintes matérielles (Qui parle/écrit et à qui ? Quels récits sont conservés et comment? Quelles relations sont établies entre divers corpus ?), fabrication à laquelle participe également le regard de cellui qui cherche à en dire quelque chose pour aujourd'hui.

3 Je m'appuierai ici principalement sur quatre lettres envoyées à Émile Zola et à Georges Saint-Paul à la fin du xix ${ }^{e}$ siècle, par un auteur anonyme ${ }^{2}$. Je m'intéresse à la manière 
dont ces lettres peuvent faire archive de diverses manières, selon des stratégies de délimitations et de lectures spécifiques. Des versions censurées de ces lettres ont été publiées au sein d'ouvrages médicaux traitant des questions sexuelles par Georges Saint-Paul (sous le pseudonyme du Dr. Laupts) en 1896, 1910 et 1930. Il s'agit probablement de la biographie sexuelle la plus célèbre de l'époque en France, rapidement devenue un classique de la littérature sur l'inversion sexuelle, et par la suite, de l'histoire de l'homosexualité européenne. Cet article propose une sorte d'homomage critique à l'auteur de ces lettres, ainsi qu'à l'historien Michael Rosenfeld, qui propose une reconstitution du manuscrit intégral des lettres dans une magnifique édition parue en 2017 sous le titre Confessions d'un homosexuel à Emile Zola. La mise en évidence par Rosenfeld des coupes et censures réalisées par Saint-Paul, ainsi que la publication de passages inédits de ces lettres, offre aujourd'hui la possibilité de nouvelles rencontres avec cette archive.

4 Je me demanderai ici de quelles façons ma position sociale de personne queer blanche européenne ainsi que les luttes politiques actuelles dans lesquelles je m'inscris - les perspectives queer anti-assimilationnistes - ou qui m'interpellent - les critiques queer décoloniales - impactent mes manières de lire ces lettres. J'aimerais articuler cette réflexion à une enquête sur "les pouvoirs affectifs de l'archive» (Cvetkovich 2003), en m'appuyant sur la transformation critique de mes relations à ces lettres, que je (re)lis depuis plusieurs années dans le cadre de mes recherches. Les émotions qui traversent mon corps de lecteurice rencontrent en effet mes questionnements méthodologiques, et orientent mon rapport à ce passé tordu (queer past), tout autant que mes capacités à y entrevoir des futurités déviantes.

5 Je propose de retracer la mutation progressive et réflexive de mes lectures de ces lettres en reconstituant trois modalités de rencontre avec cette archive, dans une tentative de formaliser différents régimes de lecture, qui se sont succédé et parfois superposés dans mon travail de recherche. Ces lectures que je nomme - après d'autres ${ }^{3}$ - histoire des minorités, lecture à rebours (against the grain) et lecture suivant le grain (along the grain) me paraissent en effet proposer des rapports politiques différents entre passé et présent, mobiliser des épistémologies du récit historique parfois incompatibles, mais aussi engager des affects et des émotions très diverses. Chercher à tordre les archives suppose d'affiner notre sens queer de l'orientation, notamment si l'on souhaite résister aux appels normatifs, homonationalistes (Puar 2007) et néocoloniaux des autoproclamées « démocraties sexuelles » européennes (Scott 2014).

\section{Histoire des minorités : sortir les fières archives du placard}

6 Une première manière de s'immerger dans l'archive queer, de la lire ou de la tordre, peut être rattachée à ce que Dipesh Chakrabarty (1998) nomme "une histoire des minorités ». Selon Chakrabarty, dans la foulée des luttes politiques menées aux ÉtatsUnis et en Europe par différents groupes opprimés dans les années 1970, une telle perspective vise à réintégrer dans le récit historique global les histoires de celleux qui en ont été longtemps exclue's. Tordre l'archive dans ce sens-là, c'est avant tout chercher à réparer une exclusion historique, comprise comme une violence épistémique redoublant les violences historiques matérielles vécues par les minorités. Ces lectures queer se placent sous le signe de la réappropriation et de la découverte des 
vies queer du passé dont il s'agit de faire entendre la réalité-véritét, pour nourrir nos propres aspirations présentes et futures. C'est cette perspective qui domine l'historiographie des lettres à Zola, ainsi que mes premières interprétations, selon une stratégie de lecture cherchant à tirer l'archive queer vers le haut, des profondeurs historiques du silence vers la visibilité et la fierté.

\section{Call me by your name ${ }^{5}$}

7 Le premier pas vers la libération est la récupération de son/notre nom. Le geste de donner un titre à l'archive révèle en effet les implications épistémologiques, politiques et affectives du pouvoir de nommer. Le choix initial, Roman d'un inverti, fait par Saint Paul avec l'accord d'Émile Zola, constitue un titre diagnostic, qui fait de l'auteur le patient zéro ${ }^{6}$ de l'inversion sexuelle innée. Le terme de roman $^{7}$ y insiste sur la division du travail entre l'inverti qui raconte et le savant qui analyse. Depuis les années 1970, la construction d'une histoire des minorités sexuelles s'attache à se réapproprier ce texte, à organiser son sauvetage épistémologique et politique de manière à l'inscrire dans l'histoire de l'émancipation des subjectivités homosexuelles ${ }^{8}$. Le choix récent de Rosenfeld, de faire de l'auteur du texte un homosexuel vise à lui faire justice et à réparer la violence épistémique médicale commise. Il s'agit de rendre sa dignité à une victime, dont la vie et l'identité ont été instrumentalisées au nom de la science. Rosenfeld précise son choix en ce sens: "L'auteur des Confessions est bien un homme qui aime d'autres hommes» (Rosenfeld $2017: 174)$ et " nous désirons honorer le courage de ce jeune homme qui refuse d'accepter la condamnation de son homosexualité " (Rosenfeld 2017 : 151). Cette idée de faire justice aux victimes de l'histoire en redressant les torts d'une archive "archiviolente» (Zimmermann 2018) et en contestant son pouvoir de nomination était aussi au cœur de mon mémoire - qui cherchait à empêcher le recouvrement de la folle sous l'homosexuel. Ma lecture était alors animée par un désir de visibilité et de guérison des traumas générés par ma plongée dans les fonds d'archives de la répression historique des pervers.

8 Le terme de Confessions fait signe vers cette même lecture rédemptrice du texte. Il s'agit de mettre l'accent sur l'authenticité d'une parole à la première personne. Rosenfeld justifie le choix du terme Confessions comme une manière d'insister sur sa valeur de «témoignage historique sur l'homosexualité » (Rosenfeld 2017: 169). Privilégier la confession au roman, c'est à la fois défendre une position épistémologique sur la possibilité de fournir les preuves de la crédibilité d'une parole, et mobiliser un ensemble d'affects lié à la découverte d'une réalité-vérité historique. Zola et Saint-Paul défendaient l'authenticité du texte à partir de leur ressenti de lecteurs: "cette confession est sincère ; elle est vraie d'une vérité que l'on sent à l'émotion » (Rosenfeld 2017 : 247). Mes premières lectures du texte en 2018 sont marquées par ces affects produits par ce qu'Arlette Farge (1989:6) nomme «l'effet de réel». Il s'agit du sentiment d'avoir soudain accès à des "traces brutes de vie", à des "morceaux de vérité à présent échoués [qui] s'étalent sous les yeux: aveuglants de netteté et de crédibilité » (Farge 1989 : 8). Si l'historienne nous rappelle que cet effet de réalité-vérité est un mirage, il n'en a pas moins des effets d'intensité et la capacité d'étancher provisoirement nos soifs d'histoires queer, d'apaiser - un peu - notre fièvre d'archives. 


\section{Archi-OUT}

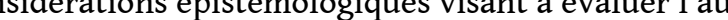
des préoccupations politiques concernant le devenir des identités et des luttes des minorités sexuelles. Pour une histoire à visée émancipatrice, ce rare témoignage de vie queer au XIX ${ }^{e}$ siècle est susceptible de représenter et d'inspirer les luttes LGBTQI+ au présent. Je célébrais dans mon mémoire ces folles militantes et combat tantes, qui «commencent à produire leur propre discours, se donnent des noms de guerre, refusent les identifications et assument leurs statuts d'anormale.aux » (Faure 2018: 143). Cette lecture de l'archive insiste sur l'autonomie conquise de cette parole invertie contre et avec les dispositifs de pouvoir - médicaux, légaux, policiers - qui la suscitent et l'encadrent. Pour Artières et Thompson (2017: 16) ces lettres apparaissent comme le point de départ d'une histoire des visibilités queer et de l'émergence de subjectivités dissidentes : « Tous ces traités scientifiques font naître, il est vrai, de nouveaux savoirs, mais ils permettent, en même temps, à de nouvelles subjectivités d'émerger ». Selon Rosenfeld encore, l'auteur des Confessions refuse de «se laisser enfermer dans un placard social» et "défend son droit à la différence" (Rosenfeld 2017: 151). Les archives sortent enfin du placard du silence et de l'oubli pour faire leurs fiers comingout dans l'histoire collective. Selon cette manière de mettre l'archive queer en lumière, la découverte d'une parole homosexuelle autonome et singulière ouvre la voie aux identifications historiques. L'excitation de trouver dans l'archive une voix qui me parle et qui parle de moi se mêle dans mon expérience de lecture à ce désir d'établir une continuité historique des existences queer. C'est cette même avidité de découverte d'un passé queer auquel il soit possible de s'identifier qui suscite la célébration de «nos ancêtres les pervers » par Pierre Hahn (2006), militant de la libération homosexuelle des années 1970 ; l'enquête historique sur les transgender warriors menée par Leslie Feinberg (1997) ; ou encore le désir d'une géologie des études queer chez Gayle Rubin, qui remonte précisément à ces premiers témoignages de pervers dans les sexologies européennes (Rubin 2011).

Cet enrôlement de l'archive dans les luttes politiques du présent peut prendre différentes formes. Christophe Girard, dans sa préface au catalogue de l'exposition Fières Archives, défend sa vision clairement assimilationniste et homonationaliste, inscrivant les archives queer dans une histoire du progrès des droits LGBT, et dans le cadre de l'État de droit libéral (Artières \& Thompson 2017 : 11). L'inverti du XIX ${ }^{e}$ siècle est placé sous la protection de l'État-Nation, présenté comme symbole de la « démocratie sexuelle » (Scott 2014) à la française. Rosenfeld insiste plus sobrement sur la manière inspirante dont l'inverti «accepte son homosexualité » car " loin d'avoir honte de sa sexualité, [il] en est fier » et l'historien considère que cette "défense pionnière et audacieuse du droit à la différence [...] résonne encore de nos jours » (Rosenfeld 2017 : 151). C'est aussi cette fierté et cette fascinante force d'affirmation de soi - mettant à distance les stigmates de la honte - qui dominaient mes premières lectures de ces lettres. La conclusion de la lettre de 1896 et la défense sadienne d'une jouissance stérile et destructrice a encore pour moi aujourd'hui une saveur toute particulière, satisfaisant pleinement mon goût d'une archive rebelle, fière et révolutionnaire: "Jouir et détruire en même temps, n'est-ce pas chose divine?» (Rosenfeld $2017: 144)$. 
11 Malgré sa rareté, l'archive queer apparaît selon cette première lecture comme « visible et préhensible » (Farge 1989: 12). Le long et minutieux travail de récupération et de reconstitution des lettres de cet inverti du $\mathrm{xIX}^{\mathrm{e}}$ siècle ouvre sur la possibilité de ressentir une archive vivante et vibrante, inspirante et militante. Ma formation théorique foucaldienne et mes recherches portant sur l'émergence des dispositifs de pouvoir autour de la sexualité me suggèrent cependant d'autres manières de tordre les archives, qui se méfient autant des tentatives d'établir des réalités-vérités historiques que de la mise en scène de voix queer authentiques. Il semble possible de s'orienter autrement dans les archives, de chercher à prendre les textes à contresens pour tenter de nager contre le courant dominant de l'histoire des minorités.

\section{Lectures à rebours (against the grain) : enquêter sur un passé en miettes}

12 «La méthodologie consistant à lire à rebours est peut-être la stratégie méthodologique clé de l'histoire queer, comme pour d'autres histoires de marginalisation » (Arondekar 2015 : 214). Cette remarque sur la lecture à rebours des archives m'a poussé à explorer et formaliser davantage certaines intuitions qui guident mon travail de recherche depuis le début de mon doctorat. Les pratiques de lecture à contre-courant au sein des études queer s'inspirent des critiques de l'historiographie coloniale menées dans les études subalternes, mais aussi de la tradition européenne de l'histoire des vaincu'e-s. Une telle lecture invite à reprendre les lettres à Zola et à Saint-Paul en les replaçant dans le champ de bataille plus large des relations entre invertis et médecins à la fin du $\mathrm{XIX}^{\mathrm{e}}$ siècle. L'archive queer se (re)compose alors à partir des traces laissées par ces luttes perdues, et la fièvre de la recherche s'apparente ici à une forme d'étourdissement provoqué par la confrontation aux violences traumatiques d'un passé en miettes.

\section{Introuvables consciences rebelles}

Une première prise de distance vis-à-vis de la perspective d'une histoire des minorités est notable dans le travail de Philippe Lejeune sur les autobiographies homosexuelles $\mathrm{du} \mathrm{XIX}^{\mathrm{e}}$ siècle. L'historien constate en effet que les paroles des invertis recueillies par les médecins "sont déjà à moitié digérées, broyées dans le texte du rapport médical » (Lejeune 1987 : 85). Ces images puissantes font écho à ma frustration ressentie au fil de mes lectures nauséeuses des traités médicaux ou policiers sur les perversions sexuelles, qui ne cessent de couper, censurer, réécrire, latiniser, ou simplement effacer les sources qui constituent les conditions de leur propre discours. Une certaine mélancolie se dégage de ces récits de vie, produits sous contrainte suivant les règles très précises de dispositifs de production d'aveux monotones et humiliants : hérédité, antécédents, état physique, fonction, facultés mentales, caractère... C'est précisément à ces sentiments de honte et de défaite, constitutifs d'une histoire des vaincu'e's, que ma première stratégie de lecture cherchait à échapper.

14 Une lecture qui insiste sur les opérations du pouvoir médical indique un autre manière de sillonner ${ }^{9}$ l'archive: non plus simplement le manuscrit reconstitué comme dépositaire d'une expérience passée, mais la relation inverti-médecin, comme dispositif de production des discours de pouvoir pathologisant les déviances sexuelles. L'édition des Confessions d'un homosexuel à Émile Zola par Rosenfeld est en ce sens un évènement 
majeur pour l'histoire queer en France, puisqu'elle propose un énorme travail de reconstitution des coupes, transformations, et réécritures opérées par le docteur Laupts. L'archive y apparaît comme un palimpseste, un monstre découpé-recollé à la Frankenstein avec ses multiples niveaux de lecture : le texte en noir pour ce qui a été publié (en 1896, 1910 et 1930), le texte en italique pour ce qui a été publié traduit en latin, le texte en gris pour ce qui a été censuré. Certains passages ont également été simplement inventés et réécrits directement par Saint-Paul, ou déplacés dans le texte. Ce qui m'intéresse dès lors, c'est moins le texte des lettres comme lieu d'expression d'une subjectivité propre que les sutures, les coupes et les censures productives, comme conditions de production des limites du texte lui-même et des effets de subjectivité qu'il produit. Cet état «broyé » de l'archive queer n'est pas exceptionnel. Jennifer Terry remarque qu'il s'agit d'une constante du rapport aux sources de l'histoire queer du xix siècle : les matériaux sont rares, très localisés, la plupart ont été détruits, et ce qui reste est toujours présenté de façon péjorative au sein de discours oppressifs sur le crime, le péché ou la maladie. Elle en tire la conclusion qu'" il ne peut $\mathrm{y}$ avoir aucune illusion de transparence ou d'accès sans médiation à l'expérience essentielle des sujets » (Terry $1991: 281)$.

La critique de l'historiographie coloniale menée par les études subalternes depuis les années 1980 insiste sur cette impossibilité de reconstituer une conscience rebelle autonome du passé, selon une approche critique qui inspire encore trop peu l'histoire des subjectivités queer occidentales. Ranajit Guha (1983), alors qu'il étudie les différents types de discours historiques tenus à propos de la révolte paysanne des santals en Inde contre les colons britanniques en 1855-56, nous rappelle que « le rebelle en tant que sujet de la rébellion n'a aucune place dans cette histoire ». Il faut renoncer selon lui à l'idée d'un accès sans médiation à la conscience insurgée, qui ne nous est accessible qu'à travers des savoirs coloniaux produits pour justifier l'existence de l'Empire. Selon Gayatri Chakravorty Spivak, la recherche d'un accès à une conscience subalterne conduit à la répétition de diverses formes d'échecs (failures) : «Le point de vue subalterne, sa volonté, sa présence, ne peuvent être que des fictions théoriques qui autorisent le projet de lecture » (Spivak $1987: 204$ ). Que devient alors l'histoire queer si elle est traversée par cette impossibilité de retrouver et de sauver nos ancêtres? Quel est le sens et l'intérêt de nos enquêtes historiques si l'on met en doute la possibilité que les pervers puissent (nous) parler (Spivak 1987)?

\section{Couper les jouissances dans l'anal-archive}

16 Mener l'enquête à contre-courant revient à tracer la carte des points localisés de conflits au sein des dispositifs de pouvoir : ici la technologie médico-policière de l'aveu qui traque les déviants sexuels en France à la fin du xix $x^{e}$ siècle. Le travail minutieux de Rosenfeld nous permet de saisir ce qui n'est pas passé à l'édition et autorise une lecture des restes produits par et abandonnés dans l'insurrection des invertis ${ }^{10}$. Non dans la perspective de reconstituer une histoire - héroïque ou pathétique - des folles mais plutôt en quête d'indices révélateurs des bordures constitutives de l'archive queer.

Lire contre le grain de l'archive revient ici littéralement à faire l'expérience d'un texte gris auquel il faut habituer les yeux ${ }^{11}$. Dans ces passages en gris - inédits dans l'édition de Rosenfeld - c'est-à-dire ces passages supprimés par Saint-Paul, on peut lire des récits d'expériences sexuelles, de découvertes érotiques et de jouissances en tous genre. 
Le caractère explicitement pornographique des lettres constitue en grande partie l'impossible excès qui ne passe pas la frontière de la publication ${ }^{12}$. Le contre discours du pervers constitue l'archive d'un ars erotica comme condition de possibilité refoulée de la scientia sexualis. Pour que la science médicale puisse traquer, à l'aide de la technologie de l'aveu, la vérité psychologique des sujets, il a fallu éliminer les traces des savoirs saisis à même l'expérience du plaisir, ces vérités du corps et de la jouissance que les lettres racontent sans cesse. Les passages censurés donnent à lire le scandale des jouissances les plus intenses. Avec le sergent d'abord "je me sentis mourir [...] je n'aurais jamais cru pouvoir jouir autant» (Rosenfeld 2017: 35). Avec le capitaine ensuite, lorsque les amants sont «anéantis » par le plaisir. Avec l'homme de l'hôtel enfin qui lui donne «les plus vives jouissances» (Rosenfeld 2017 : 102). C'est toujours cette jouissance qui conclut la dernière lettre : «J'ai joui et fait jouir de moi de mille façons» (Rosenfeld 2017: 139). À quoi ressemblerait l'histoire de la sexualité si les jouissances des pervers n'avaient pas été systématiquement recouvertes par les souffrances des invertis? Cette jouissance circule pourtant sur tout le corps érotisé, et fait l'objet de patients apprentissages :

Il me suçait pendant ce temps les oreilles, introduisait sa langue dans ma bouche et parcourait de ses mains tout mon corps. Il me disait d'une voix brisée les plus douces paroles et les mots les plus insensés. [...] Il disait que tous les membres devaient contribuer au plaisir et mettait à exécution ce qu'il disait. Il inventait les positions les plus étranges, les mouvements alternés et cadencés, les sauts et les torsions les plus extraordinaires, je ne vous dirais pas tout ce qu'il m'apprit. (Rosenfeld 2017 : 45)

18 J'aimerais évoquer une jouissance en particulier - largement refoulée par l'archive médicale - dans cette exploration des plaisirs sensuels, celle de la pénétration anale. Une carte postale envoyée à Zola annonce un sentiment de joie triomphale lorsque son anus est pénétré pour la première fois : "Ce qui devait advenir est advenu. J'en garde encore le plus délicieux souvenir et suis parfaitement heureux ce matin, je vous assure. Je le crierais sur les toits. Là où tous avaient échoué, il a réussi !» (Rosenfeld 2017 : 65). Cette extase anale m'apparaît comme le cœur d'une possible lecture vengeresse (Terry 1991 : 289) de l'archive médicale des perversions sexuelles. Il faudrait replacer l'anus et l'extase du corps pénétré au centre de la lecture, contre une histoire officielle dédiée à son effacement systématique. Laupts coupe tout ce qu'il peut - mais le sexe anal laisse toujours quelques traces. Des premières tentatives de pénétration avec le capitaine, le médecin ne garde que la douleur et l'échec, il coupe les pratiques de dilatation et l'usage d'un tube de cold cream pour la lubrification. De l'aventure de l'hôtel, il supprime plus de dix pages. Dans ces passages effacés pour la publication, l'enculé esquisse les fragments d'une contre anal-archive extatique.

L'aventure de l'hôtel ne fut que passagère - quoique hélas ! Elle laissa plus de traces que les autres qui l'avaient précédée. En m'ouvrant de nouveaux horizons elle me fit devenir ce que je suis et ce que je ne cesserais plus d'être. (Rosenfeld 2017 : 87)

Une lecture à rebrousse-poil des lettres esquisse une mémoire des vaincu'e-s en train de perdre la bataille, et permet d'entrevoir l'effacement quasi-systématique des jouissances dans les archives de la sexologie française de la fin du XIX ${ }^{e}$ siècle. Lire contre le grain, c'est emprunter le paradigme indiciaire de Ginzburg (1980), à la recherche d'indices, de traces et de pistes plus que d'évidentes réalités-vérités. C'est 
tenter de saisir des effets de subjectivités dans le jeu incessant des rapports de force entre médecins et pervers. En lisant à rebours et dans le gris, je rencontre des anéantissements et des extases anales fugaces plus que des subjectivités fières. Sur le plan affectif, la perte, l'amertume, le deuil, le regret ou la mélancolie prennent alors la place des identifications fières et inspirantes. Si ces émotions semblent à première vue «mauvaises pour la politique» (Love $2007: 12$ ), elles resituent l'histoire queer et ses effacements systématiques dans celle des parias de l'histoire. Elles ouvrent la voie à des alliances entre les existences infâmes, censurées et marginalisées dans les récits historiques dominants.

\section{Lectures suivant le grain (along the grain) : hériter de nœuds obstinés}

20 J'ai pourtant la sensation que ces deux premières lectures - histoire des minorités et lecture à rebours - n'épuisent pas la réception actuelle - émotionnelle et politique de ces lettres; et qu'il est possible de faire autre chose de cette rencontre étrange avec les mots que cette folle du XIX ${ }^{e}$ siècle a décidé de confier à Zola. Ces manières de lire me conduisent en effet à laisser de côté plusieurs " nœuds obstinés » (Chakrabarty 1998 : 22) de l'archive. La féminité embrouillée et contradictoire de l'auteur des lettres, ou encore l'histoire raciale de sa généalogie familiale ne trouvent pas facilement leur place dans ces stratégies d'interprétations libératrices, que celles-ci soient dédiées à reconstruire les subjectivités homosexuelles du passé ou à tracer les contours des dispositifs de pouvoir qui les produisent. Je me trouve donc à la recherche d'une autre manière d'analyser ces lettres, qui permettrait de maintenir l'intranquillité ${ }^{13}$ de la rencontre avec l'archive queer. Suivant les traces des réflexions épistémologiques de Ann Laura Stoler (2019), j'aimerais esquisser quelques pistes de ce que pourraient être des « lectures suivant le grain » (along the grain).

21 Selon Chakrabarty, le passé de celleux qui ont été minorisée-s, considéré'e·s comme incapables de rationalité, ne peut pas être absorbé dans le récit historique dominant des démocraties libérales : « le récit d'un fou, ce n'est pas de l'histoire » $(1998: 16)$. Les passés subalternes sont définis par leur résistance obstinée à l'historicisation, et mettent en crise les catégories de l'histoire elle-même. Le passé subalterne des pervers aurait ainsi le pouvoir de fracturer le présent et de déstabiliser nos catégories d'entendement de nous-même. Halberstam (1998: 53) développe la proposition d'un " présentisme pervers » comme possibilité d'entrer en relation avec les «énigmes du passé », qui me semble pouvoir être articulé à cette préoccupation des études subalternes. Sa lecture des «masculinités féminines » du XIX siècle renonce à projeter nos catégories de genre et de sexualité pour décrire les expériences du passé et contribue à mettre en crise les identités queer contemporaines. Que devient notre lecture des lettres à Zola si l'on cherche à lire les identifications de genre et de sexualité de l'auteur en tenant à distance ce que l'on pense savoir de l'homosexualité masculine dite moderne, ou des transidentités contemporaines? Quels seraient également les effets sur nous-mêmes d'une lecture qui s'attacherait à suivre la présence envahissante des catégories raciales et coloniales dans ces lettres, esquissant la position paradoxale des invertis européens, à la fois pervers et civilisés?

Lire en suivant le grain des archives permet de prendre en charge le sentiment d'altérité insurmontable qui se dégage de ces lettres et de faire une place aux éléments 
les plus déstabilisants pour ma lecture du récit - les identifications genrées et raciales de l'auteur. Il faut peut-être partir du principe que l'on ne connaît pas déjà ces textes, et tenter de se rendre sensible à leur relief : «Lire l'archive suivant le grain ${ }^{14}$ (along the grain) tire notre sensibilité moins vers la texture homogène de l'archive que vers son relief, vers la surface rugueuse qui nuance sa couleur et modèle sa forme " (Stoler 2019 : 89). L'archive ici n'est plus considérée comme un objet perdu-retrouvé selon une lecture préoccupée par les silences et les résistances, les absences et les révoltes, mais plutôt comme un sujet ambigu et récalcitrant, avec sa forme et son contexte propre, avec ses présences inattendues qui sollicitent des stratégies de lecture imaginatives. Plutôt que de traquer les traces enfouies des batailles menées par les pervers contre le dispositif médical de la sexualité, cette manière de lire permet de s'intéresser aux rationalités discursives qui permettent de dire la sexualité, le genre, la race ou l'empire dans des récits de soi souvent contradictoires et contingents.

\section{Les drôles de genres des invertis}

À propos des identifications de genre de l'auteur des lettres, les limites de mes deux premières stratégies de lecture m'apparaissent clairement. L'histoire des minorités aurait tendance à dire : « contrairement au stigmate de l'efféminement et de l'inversion projetée sur lui par Saint-Paul, l'auteur de ces lettres est un homosexuel, c'est-à-dire un homme qui aime les hommes ». La contre-histoire déviante de son côté aurait tendance à dire : « Les lettres témoignent des dispositifs de capture disciplinaire qui fabriquent le pervers, sans réussir cependant à effacer totalement les traces de leurs censures productives». Une lecture qui caresserait l'archive dans le sens du poil, tout en assumant l'impossibilité d'accéder aux formes d'altérités subjectives du passé, permet d'imaginer la forme d'une variance de genre spécifique, et rend possibles «les multiples histoires de sujets non normatifs» (Halberstam 1998: 57). Mobilisant les affects de la curiosité, de la perplexité et de la confusion, cette approche relève aussi d'une «fabulation critique » (Hartman $2008: 11$ ) qui fait l'épreuve de la manière dont ces passés queer résistent aux récits du présent.

Contre le docteur Laupts qui voit dans son cas le prototype parfait de l'inverti-né féminiforme, l'auteur des lettres fait clairement savoir qu'il n'a rien d'efféminé. Il me semble pourtant difficile de le considérer simplement comme un homme - comme le font la plupart des commentateurs contemporains. Ces revendications de masculinité entrent en effet en contradiction avec l'abondance des identifications féminines qu'il retrace depuis son enfance, son amour des figures féminines de l'histoire et de la littérature - de Marie-Antoinette à la Vierge Marie, le plaisir qu'il prend à se glisser dans les robes de sa mère, ou encore les regrets clairement formulés de ne pas être né femme. Il faut cependant aussitôt remarquer que cette féminité tant désirée est ellemême difficile à (re)connaître et à cerner. Il s'agit d'une féminité qui s'exprime sur le registre du désir sexuel et des fantasmes érotiques, plus que sur celui de l'identité psychique. L'auteur qualifie en effet de « désir féminin » sa position sexuelle vis-à-vis des hommes virils de sa vie et de ses rêves : «Je me passionnais véritablement pour lui et je me plaisais à me figurer d'être Andromaque, pour pouvoir tenir dans mes bras le héros bardé de fer " (Rosenfeld $2017: 20$ ). Pourtant après une nuit de passion avec le sergent, l'expérience du plaisir et la confirmation de sa désirabilité le conduisent à aimer être autre chose qu'une femme: "Je ne désirais plus être une femme, car je 
trouvais cette passion terrible bien plus savoureuse et plaisante, supérieure à ce que peut offrir l'amour connu » (Rosenfeld $2017: 36$ ).

Et lorsque ces identifications de genre déviantes et non normatives s'expriment plus clairement sur le registre de l'identité, de nouvelles déroutes attendent nos/mes désirs de rencontrer des figures queer du passé clairement reconnaissables ${ }^{15}$. Le manque de catégories pour se dire requiert d'opérer quelques torsions au cœur de la différence sexuelle: homme peut-être, mais alors androgyne comme Ganymède; femme probablement, mais alors reine comme Marie-Antoinette. Cette difficulté à y voir clair s'aggrave encore lorsque l'auteur tente de définir la nature de son âme, le plus souvent en ayant recours au registre du mélange, du monstrueux, de ce qui qui excède les cadres et les catégories: "un être exceptionnel et fantastique ", "une âme monstrueuse », un «être bizarre» (Rosenfeld $2017: 129)$. Comment faire l'histoire de celui qui présente son existence comme située à la limite de l'intelligible? On peut seulement tenter de suivre le fil de ces (dés)identifications et des paysages complexes qu'elles permettent d'imaginer. Si l'auteur des lettres décrit son corps dans le registre des beautés masculines de l'éphèbe, son esprit - royaliste de conviction - est du côté des héroïnes et des reines : «Homme et charmant par le corps, je possède l'esprit, le charme et tous les goûts des plus délicieuses femmes; je puis donc triompher quelquefois par les dons réunis des deux sexes » (Rosenfeld 2017: 49). Ni le corps ni l'esprit ne disent pourtant la vérité ou la nature de son être, qui semble assumer un mélange de masculinité androgyne et de féminité puissante. Les tentatives ou les efforts de l'auteur pour réduire cette complexité qui déjoue les binarités de genre ne le (nous) mènent nulle part : «Je me contenterai désormais d'être ce que je suis : un être bizarre, ni homme ni femme, ou plutôt homme et femme à la fois » (Rosenfeld 2017 : 129).

26 Ces lettres caressées dans le sens du poil laissent alors entrevoir une histoire des féminités masculines françaises au xix ${ }^{\mathrm{e}}$ siècle. Ce serait l'histoire des variations de genre spécifiques et multiples incarnées par les tantes, les mignons, les pédérastes, les invertis, les pervers et les travestis ${ }^{16}$, une histoire pleine de surprises et de complexité qui exploreraient les intrications historiques du genre et de la sexualité, une histoire que les catégories d'homosexualité ou de transidentité ne suffisent probablement pas à délimiter.

\section{Pour une histoire raciale de la sexualité}

L'orientation de mes recherches présentes vers une généalogie coloniale de l'homosexualité européenne m'amène à repérer la présence des préoccupations raciales et impériales dans ces lettres à Zola, dans l'ensemble des travaux de Saint-Paul et dans les discours de la sexologie française en général. La prolifération des discours racialisants, eugénistes et impérialistes semble être la norme plutôt que l'exception dans l'histoire de la sexualité européenne, qui se constitue au moment même où la France étend drastiquement son empire colonial, et qui s'appuie et repose notamment sur la médecine coloniale, militaire ou navale, source de production de récits sexoraciaux depuis tous les recoins de l'Empire. À l'encontre d'une histoire de l'homosexualité lavée plus blanche que blanche ${ }^{17}$, rejetant le récit des violences coloniales et leurs épistémologies racialisantes aux poubelles de l'histoire queer (Bauer 2017) ; mais aussi à distance d'une recherche des opérations d'effacements des rapports de force impérialistes - le thème de la race est bien plutôt omniprésent dans les lettres 
- je m'intéresse ici à l'écriture d'une subjectivation paradoxale, celle des «pervers civilisés » dans la France impériale.

Le rôle de ces lettres dans le développement de discours impérialistes sur les perversions sexuelles apparaît comme central si l'on s'intéresse à ce qu'en fait SaintPaul dans ses écrits sur la sexualité, et à leur inscription dans la sexologie coloniale européenne de la fin du XIX ${ }^{e}$ siècle. La carrière et les convictions coloniales de SaintPaul sont peu remarquées par les commentateurs des lettres et de ses travaux ${ }^{18}$. L'intrication de ses préoccupations sexuelles et coloniales sont pourtant explicitement lisibles jusque dans ses textes les plus classiquement sexologiques. La seconde édition de l'œuvre de Saint-Paul en 1910, est en effet augmentée d'un sixième chapitre et d'une conclusion résumant les thèses principales du traité, qui mentionne clairement la "contamination coloniale» comme l'une des causes de la propagation de l'homosexualité en France: " en France comme en Angleterre, des gens s'investissent aux colonies. Revenu du Tonkin, tel français qui était parti hétérosexuel devient à son retour un foyer de corruption sexuelle» (Laupts 1910: 365). L'inversion sexuelle européenne constitue pour Saint-Paul - comme pour tant d'autres de ses confrères notamment au sein de la revue Archives d'anthropologie criminelle - la menace d'une contagion racialisée d'un vice qui déstabilise les frontières et la vitalité biologique du corps national. Les conclusions de l'ouvrage exposent directement ce nœud racesexualité, puisque l'homosexualité ne s'y dit qu'à travers la colonialité (Lugones 2008). Son statut d'exception en France, où elle est synonyme d'individualité souffrante, honteuse et isolée, garantit le caractère d'une homosexualité blanche et presque civilisée ; qui s'oppose aux perversions endémiques, habituelles et collectives observées dans les populations colonisées ${ }^{19}$. Les discours sur les perversions sexuelles dans la sexologie française apparaissent alors indissociables de préoccupations raciales quant aux possibles contaminations de la race blanche au contact des populations colonisées. Le thème de la dégénérescence de la race blanche d'une part, et les idéologies coloniales évolutionnistes et eugénistes d'autre part, constituent en effet, dans la seconde moitié du xIx siècle en Europe, l'une des matrices de fabrication de la sexualité.

Une lecture suivant le fil du texte échappe en effet difficilement à cette saturation du thème du "croisement des races", qui occupe littéralement la moitié de la longue lettre à Saint-Paul de 1896. L'auteur se lance dans une vaste généalogie familiale, avec l'objectif assumé de produire la démonstration de l'origine de sa nature déviante. La race n'est pas un élément marginal du discours sur la sexualité mais l'une des raisons centrales permettant d'expliquer - sur plus de dix pages des lettres - l'émergence de l'inversion chez l'auteur. Du côté paternel, la famille est espagnole, établie en Italie et fascinée par les mœurs anglaises. C'est du côté maternel que les choses se compliquent. Et surtout avec les grands-parents maternels, qui sont l'objet dans les lettres de descriptions sexo-raciales situées dans le droit fil de l'orientalisme sexuel dominant le XIX siècle (Saïd 2005), associant systématiquement les perversions sexuelles aux populations colonisées. Le portrait animalisé du grand-père turc, qualifié de singe avec une "bouche lippue », le situe clairement hors de la blanchité européenne. Mais c'est surtout la grand-mère maternelle qui occupe une place centrale dans le récit, selon un portrait qui fourmille des détails de sa perversion orientale. Nommée Jézabel ${ }^{20}$ par l'auteur, elle a "les joues fardées et les yeux allongés », mais aussi " une intelligence et un esprit diaboliques ». Elle ne se remarie pas après la mort de son mari « pour pouvoir 
se livrer à ses instincts désordonnés ", et vit dans son appartement entourée par des « idoles égyptiennes avec des museaux de bêtes ». Elle a « le type assyrien ou égyptien plutôt que juif $»^{21}$, et « des cheveux noirs et lustrés » (Rosenfeld 2017 : 111-115).

L'auteur des lettres tient quant à lui - dans un premier temps - à affirmer sa blanchité et son caractère anglais : «je suis tout aryen et la structure de mon corps rappelle les plus charmantes statues de la Grèce » (Rosenfeld 2017 : 119). Aryen et blanc comme un pédéraste d'Athènes, et non oriental et sombre comme un pervers de Sodome. C'est pourtant rapidement par cette hérédité raciale du désordre des instincts qu'il donne l'explication de toute sa personnalité : "L'Occident et l'Orient avec leurs sangs et leurs civilisations si diverses se sont trouvés réunis en moi - leur choc ne pourrait-il pas avoir déterminé mon étrange personnalité ?» (Rosenfeld 2017 : 129). Tout en insistant sur son identification comme aryen civilisé, l'auteur des lettres reconnait les origines orientales de son étrange sexualité et fantasme sa nature - indissociablement sexuelle et raciale -, de primitif de l'intérieur. Il se dit « ataviste », et croit à la résurgence de caractères et traits du passé dans un présent civilisé. Dans une logique évolutionniste et coloniale, ces traits dits " primitifs » renvoient aussi bien au passé de l'Europe qu'au présent des populations colonisées. Les pervers sexuels comme les criminels sont ainsi clairement positionnés comme "de véritables sauvages au milieu de la brillante civilisation européenne » (Lombroso $1887: 32$ ). Une lecture suivant le grain me permet de travailler avec les tensions des identifications raciales et des discours racistes évolutionnistes de l'auteur des lettres, tout autant qu'avec les registres irrationnels de l'imaginaire et du fantasme.

31 Ce régime de lecture des lettres nous permet d'entrevoir une histoire du dispositif moderne/colonial de la sexualité (Lugones 2008), avec ses politiques visant à «faire vivre » la race blanche européenne et sa puissance civilisatrice, et à " laisser mourir » celles et ceux supposée's menacer sa suprématie ${ }^{22}$. Chercher à se repérer dans le foisonnement indiscipliné des nœuds de la race et de la sexualité au fil des lettres nous a conduit à délimiter la position des invertis européens dans ce dispositif, une position liminale et paradoxale, entre civilité altérée et dégénérescence raffinée.

\section{Conclusion}

"Les archives queer doivent traverser les frontières dans toutes les directions" (Nestle 2015: 241). Joan Nestle, activiste aux Lesbian Herstory Archives, insiste sur l'importance actuelle de tordre les archives dans tous les sens pour résister à l'inclusion des histoires LGBTI+ dans certains agendas nationalistes et impérialistes aux États-Unis et en Europe. Tordre (queer) l'archive permet d'éprouver sa résistance actuelle aux normativités identitaires et à leurs cloisonnements essentialistes, mais aussi d'explorer son imbrication avec les histoires coloniales et capitalistes, par-delà les séparations disciplinaires et de champs d'études. Queer (tordre) prend le sens d'un geste politique de résistance, cherchant ici à saboter la montée des discours et politiques homonationalistes en Europe. Queer désigne enfin une orientation affective, au départ d'une "nouvelle romance avec l'archive", cherchant à "la saisir sans la fixer" (Arondekar 2009: 1-4) pour fabuler de manière critique des communautés perdues et pourtant si vivantes. 


\section{BIBLIOGRAPHIE}

ARONDEKAR, Anjali. 2009. For the record, On Sexuality and the Colonial Archive in India. Durham \& London : Duke University Press.

ARONDEKAR, Anjali, CVETKOVICH, Ann, HANDARDT, Christina B., KUNZEL, Regina, NYONG'O, Tavia, RODRIGUEZ, Juana María \& STRYKER, Susan. 2015. « Queering Archives, A Roundtable Discussion » in Radical History Review 122. Durham : Duke University Press.

ARTIERE, Philippe \& THOMPSON, Clive. 2017. Fières archives, documents et images autobiographiques d'homosexuels « fin de siècle ». Neuilly : Atlande.

BACHETTA, Paola. 2018. « Les Forces Transformatives d'Archives des Queers Racisé.e.s », intervention à l'occasion des Ateliers-Débats pour la création d'un centre d'archives LGBTQI, Paris, [en ligne], consulté le 30 avril 2021. URL : https://friction-magazine.fr/re-presence-lesforces-transformatives-darchives-de-queers-racise-e-s/

BAUER, Heike. 2017. The Hirschfeld archives : violence, death, and modern queer culture. Philadelphia : Temple University Press.

BURTON, Richard. 2009. Les mille et une nuit ; la zone sotadique. Traduction de Jean Claude Bouvard. Lille : Gaykitschcamp.

CASES REBELLES (Collectif). 2021. Afro Trans. Nantes : Éditions Cases Rebelles.

CHAKRABARTY, Dipesh. 1998. « Minority histories, subaltern pasts » Postcolonial Studies : 1(1) : 15-29.

CHOW, Rey. 2002. The Protestant Ethnic and the spirit of capitalism. New York : Columbia University Press.

COHEN, Joni Alizah. 2019. « L'éradication des “abstractions talmudiques” : l'antisémitisme, la transmisogynie et le projet nazi ». Contretemps [En ligne], hors série. Traduction de Sophie Coudray. consulté le 8 mai 2021. URL : https://www.contretemps.eu/antisemitismetransmisogynie-nazisme/

CVETKOVICH, Anne. 2003. An archive of feelings, Trauma, Sexuality, and Lesbian Public Cultures. Durham : Duke University Press.

FARGE, Arlette. 1997 [1989]. Le goût de l'archive. Paris : Le Seuil.

FAURE, Ruby. 2018. Féminité et barbarie, émergence de l'homosexualité masculine blanche en France et en Europe, 1870-1914. Mémoire d'études de genre : Université Paris 8.

FEINBERG, Leslie. 1997. Transgender warriors, making history from Joan of Arc to Dennis Rodman. Boston : Beacon Press.

FOUCAULT, Michel. 2003. Le Pouvoir Psychiatrique (1973-1974), Cours au Collège de France, Leçon du 6 février 1974. Paris : Le Seuil.

FOUCAULT, Michel. 1994 [1971]. « Nietzsche, la généalogie et l'histoire », in Dits et Ecrits 1, FOUCAULT, Michel. Paris : Gallimard : 1004-1024.

GINZBURG, Carlo. 1980. « Signes, traces, pistes. Racines d'un paradigme de l'indice » Le Débat 6 : $3-44$.

GROJNOWSKY, Daniel (édition établie par). 2007. Confessions d'un inverti-né. Paris : José Corti. 
GUHA, Ranajit. 1983. «The Prose of Counter Insurgency ", in Selected Subaltern Studies, GUHA Ranajit and SPIVAK Gayatri Chakravorty (eds). Oxford : Oxford University Press, 45-86.

HAHN, Pierre. 2006 [1979]. Nos ancêtres les pervers, La vie des homosexuels sous le seconde empire. Saint-Martin-de-Londres : H\&O éditions.

HALBERSTAM, Jack. 1998. Female masculinities. Durham, London : Duke University Press.

HARTMAN, Saidiya. 2008. «Venus in Two Acts » Small Axe 26 : 1-14.

LOVE, Heather. 2007. Feeling Backward, Loss and the Politics of Queer History. Cambridge : Harvard University Press.

KEBABZA, Horia. 2006. " "L'universel lave-t-il plus blanc ?" : "Race”, racisme et système de privilèges » Les cahiers du CEDREF [En ligne], 14, consulté le 15 novembre 2021. URL : http:// journals.openedition.org/cedref/428

LAUPTS Dr. (SAINT-PAUL Georges). 1896. Tares et poisons, perversion et perversité sexuelle. Paris : G. Carré.

LAUPTS Dr. (SAINT-PAUL Georges). 1910. L'Homosexualité et les Types homosexuels. Paris : Vigot frères.

LEGLUDIC, Henri. 1896. Notes et observations de médecine légale, attentats aux mœurs. Paris : Georges Masson Editeur.

LEJEUNE, Philippe. 1987. « Autobiographie et homosexualité en France au XIX ${ }^{\mathrm{e}}$ siècle » Romantisme $56: 79-94$.

LOMBROSO, Cesare. 1887. L'homme criminel. Etude anthropologique et psychiatrique. Traduction de Albert Régnet et Georges Bournet. Paris : Félix Alcan Editeur.

LUGONES, Maria. 2008. «Colonialidad y genero » Tabula Rasa [En ligne], 9, consulté le 30 avril 2021. URL : https://www.revistatabularasa.org/numero-9/05lugones.pdf

MACE, Gustave. 1889. Mes lundis en prison. Paris : Charpentier.

MEYER-PLANTUREUX, Chantal. 2019. Antisémitisme et homophobie, Clichés en scène et à l'écran, XIXe $-\mathrm{XX}$ siècles. Paris : CNRS Editions.

MURAT, Laure. 2006. Une Histoire culturelle $d u$ « troisième sexe ». Paris : Fayard.

NESTLE, Joan. 2015. « Who Were We to Do Such a Thing? Grassroots Necessities, Grassroots Dreaming » Radical History Review $122: 233-242$.

PENISTON, William A. \& HERBER, Nancy. 2012 [2007]. Bougres de vies (Queer lives). Huits homosexuels du XIX ${ }^{e}$ siècle se racontent. Traduction de Marie Clément. Cassaniouze : ErosOnyx Éditions.

PUAR, Jasbir. 2007. Terrorist Assemblages : Homonationalism in Queer Times. Durham : Duke University Press.

ROSARIO, Vernon. 2000. L'irrésistible ascension du pervers, entre littérature et psychiatrie, Traduction de Guy le Gaufey. Paris : Éditions Epel.

ROSENFELD, Michael (textes réunis et présentés par). 2017. Confessions d'un homosexuel à Emile Zola. Paris : Nouvelles éditions Place.

RUBIN, Gayle. 2011. Deviations : a Gayle Rubin Reader. Durham \& London : Duke University Press.

SAÏD, Edward W. 2005. L'Orientalisme. Traduction de Catherine Malamoud. Paris : Le Seuil. 
SCOTT, Joan. 2014. «Emancipation et égalité : une généalogie critique », intervention au colloque Penser l'émancipation [En ligne], consulté le 30 avril 2021. URL : https://www.contretemps.eu/ emancipation-et-egalite-une-genealogie-critique/

SPIVAK, Gayatri Chakravarty. 1987. «Subaltern Studies : Deconstructing historiography », in In other worlds, essays in cultural politics, SPIVAK, Gayatri Chakravarty. New York \& London: Methuen, pp.197-221.

SPIVAK, Gayatri Chakravarty. 1988. "Can the Subaltern Speak? ", in Marxism and the interpretation of Culture, NELSON, Cary \& GROSSBERG, Larry (eds.). Champaign : University of Illinois Press.

STOLER, Ann Laura. 2019 [2009]. Au cœur de l'archive coloniale. Questions de méthode, Traduction de Christophe Jaquet et Joséphine Gros. Paris : Éditions de l'EHESS.

SYMONDS, John Addington. 1883. A Problem in Greek Ethics. Londres : Edition privée.

TERRY, Jennifer. 1991. « Theorizing deviant historiography » Differences: A Journal of Feminist Cultural Studies 3(2) : 53-71.

ZIMMERMANN, Qiu. 2018. Résister aux oublis et aux silences : Les projets d'archives LGBTQI+ en France. Mémoire spécialité « Genre, politique et sexualité ». Paris : EHESS.

\section{NOTES}

1. Ce sont les numéros 120 : «Queering Archives: Historical Unravelings », paru en 2014, et 122 : "Queering Archives: Intimate Tracings », paru en 2015.

2. Dans la suite du texte, je me réfèrerai à cette archive sous la mention des lettres à Zola et à Saint-Paul. Il s'agit de deux lettres et d'une carte postale adressées à Zola en 1889 (pour un total de 51 pages dans l'édition de Rosenfeld 2017), et d'une lettre adressée à Saint-Paul en 1896 (40 pages dans l'édition de Rosenfeld $i d$.). On sait peu de choses sur l'auteur, hormis qu'il s'agit d'un jeune aristocrate italien, qui dit avoir 23 ans lorsqu'il écrit la première lettre à Zola, en 1889 .

3. J'emprunte ces expressions respectivement à Chakrabarty (1998), Terry (1991) et Stoler (2009).

4. Cet effet de "réalité-vérité" au contact de l'archive renvoie à la sensation de saisir des existences historiques dans leur matérialité ( « c'est comme cela que $X$ et $Y$ vivaient »), mais aussi dans leur signification ("c'est comme cela que $\mathrm{X}$ ou $\mathrm{Y}$ se pensaient»). Les discussions épistémologiques sur la notion de fait historique, leurs parts de vérité et de fiction sont au-delà du champ de discussion de cet article, mais cette " histoire des minorités " prend en général le parti pris de l'objectivité historique, et parfois d'un certain essentialisme, puisqu'il s'agit de retrouver les « homosexuels » du XIxe siècle.

5. Selon le titre de la chanson Montero (Call me by your name) par Lil Nas X, 2021.

6. D'autres récits ou témoignages existent avant, mais ce texte est le plus long, le plus complet et le plus dense publié en langue française, aux côtés d'un autre publié par Legludic (1896) sous le titre Confidences et aveux d'un parisien, La comtesse, Paris, 1850-1861. L'inversion sexuelle innée ou congénitale (perversion) est alors distinguée de l'inversion occasionnelle, liée aux influences du milieu (perversité), et si l'auteur des lettres constitue le prototype de la première catégorie, pour Saint-Paul, Oscar Wilde est l'incarnation de la seconde.

7. Rosario remarque que le terme de roman était utilisé dans la sexologie du xixe siècle dans le cas des récits des hystériques: «le terme roman faisait la différence entre une confession à la première personne et les écrits médicaux "objectifs qui l'entouraient". Les romans des hystériques avaient été franchement dénoncés comme trompeurs » (Rosario $2000: 66$ ). S'il s'agit d'un roman, la vérité du texte réside alors dans les mots des experts capables de démêler la part de l'authentique et du vécu, et de censurer ou réécrire les fabulations d'un sujet vaniteux. 
8. Avant l'édition de 2017, les lettres sont notamment éditées successivement par Pierre Hahn (1979), Grojnowski (2007) et Erber \& Peninston (2012).

9. J'emprunte cette expression à Michaëla Danjé, qui fouille l'archive coloniale pour hériter des traces historiques des constructions variées du genre et des sexualités sur le continent africain, et faire place au trouble que ces histoires suscitent au présent dans les récits blancs et occidentaux des transidentités (Collectif Cases Rebelles 2021).

10. Il s'agit de proposer un écho avec « l'insurrection des hystériques » pour laquelle Foucault propose un cadre d'analyse dont je m'inspire ici dans le cadre de l'histoire de l'inversion sexuelle : «J'essaierai d'analyser cela non pas du tout en termes d'histoire des hystériques, ni non plus en termes de connaissances psychiatriques prises sur les hystériques, mais de l'analyser en termes de bataille, d'affrontement, d'enveloppement réciproque, de disposition de pièges en miroir, d'investissement et de contre-investissement, de tentative de prise de contrôle entre les médecins et les hystériques. » $(2003: 310)$.

11. Le choix d'édition de Rosenfeld, de faire apparaître en gris les passages censurés, fait écho à la définition que Foucault donne de la généalogie : « La généalogie est grise ; elle est méticuleuse et patiemment documentaire. Elle travaille sur des parchemins embrouillés, grattés, plusieurs fois récrits. » (Foucault $1994: 1004)$.

12. Ce ne sont pas les seules coupes et censures effectuées au cours du processus de production de la parole invertie dans l'archive médicale, mais celles-ci sont systématiques et massives. Elles répondent à la fois aux exigences morales - classiques à l'époque - de la publication et aux nécessités de façonner une subjectivité souffrante et honteuse.

13. Je suis redevable à Michaëla Danjé pour cette formulation de ce que je comprends comme un engagement éthique et politique dans le rapport à l'archive : maintenir l'intranquillité c'est faire exister des flous identitaires, des troubles définitionnels, ne pas chercher à résoudre ou à intégrer les marges sous le rouleau compresseur de l'universalité (Collectif Cases Rebelles 2021).

14. Suivant la proposition de ma directrice de thèse Marie-Dominique Garnier, je préfère traduire along the grain par « suivant le grain » plutôt que par le trop straight « dans le droit fil » proposé par Christophe Jacquet et Joséphine Gros, pour la traduction française de ce texte publiée en 2019.

15. Heike Bauer attire notre attention sur ce brouillage de la perception symptomatique de l'histoire queer, en peignant un ange queer de l'histoire avec du sable plein les yeux: «Contrairement à la figure aux yeux ouverts du progrès historique, si célèbre dans la lecture de Walter Benjamin de l'Angelus Novus de Paul Klee, l'ange queer de l'histoire a la vue obscurcie par le sable de l'expérience. » (Bauer 2017 : 9).

16. Laure Murat saisit quelques unes de ces narrations dans son histoire culturelle du troisième sexe (Murat 2013).

17. J'emprunte cette métaphore à Horia Kebabza (2006).

18. Le médecin est pourtant en poste à Alger pour un an à partir de 1895, puis en Tunisie pendant cinq ans, et la rédaction de son œuvre Tares et Poisons se fait entièrement depuis la colonie. Sous le pseudonyme Georges Espé de Metz, il publie trois ouvrages qui portent explicitement sur les politiques de gouvernementalité coloniale, dédiés aux manières de maintenir et renforcer la domination française sur le peuple algérien : Souvenirs du Maroc et de l'Algérie en 1904, Vers l'empire en 1913 et Par les colons, en 1914.

19. On retrouve cette géographie coloniale des perversions dans les écrits de Richard Burton (2009) ou de Symonds (1883). La réédition du traité de Saint-Paul en 1910 fait également apparaître en annexe "L'opinion d'un musulman algérien sur l'homosexualité », qui vient confirmer l'indulgence des « indigènes » pour l'homosexualité : « Il est certain qu'en Algérie et en Tunisie, le Naiec n'est pas un être exceptionnel » (Rosenfeld 2017 : 432).

20. Jézabel est une princesse phénicienne, fille d'Ithobaal Ier, roi de Tyr et de Sidon. Elle est l'épouse du roi d'Israël Achab qui règne de -874 à -853 , et présentée dans la bible comme une 
épouse malfaisante, qui encourage notamment le culte de Baal. Elle incarne dans cette lettre la figure orientaliste de la femme étrangère puissante et corruptrice.

21. La co-construction de l'antisémitisme et de l'homophobie ou de la transmisogynie au xIxe siècle structure clairement le dispositif moderne de la sexualité, comme ce récit autobiographique (Cohen 2019, Meyer-Plantureux 2019).

22. Rey Chow (2002) propose ainsi de faire pivoter le tome 1 de l'Histoire de la sexualité de Foucault sur son chapitre 5, introduisant les concepts de «biopolitique» et de «régulation des populations ", plutôt que de s'appuyer principalement sur les régimes disciplinaires de l'identité, comme le font la plupart des études sur la sexualité. Une telle perspective permettrait de remettre au centre de l'analyse les dimensions coloniales et racialisantes des discours sexuels produits dans les formations impériales européennes du xIXe siècle.

\section{RÉSUMÉS}

Les allers-retours entre questionnements épistémologiques, positionnements politiques et expériences affectives me permettent de déployer plusieurs directions du projet de tordre (queering) les archives. L'édition par Michael Rosenfeld de lettres envoyées par un inverti à Émile Zola et au docteur Saint-Paul à la fin du XIX siècle est dans cet article l'occasion de repérer quelques transformations de mon rapport au passé queer, en distinguant trois régimes de lecture de ces lettres que j'ai développés au fil de mes recherches. Le projet d'une histoire des minorités sexuelles vise la découverte et le sauvetage des voix queer du passé, et cherche à leur rendre justice pour inspirer les luttes de libération du présent. Une lecture à rebours ou à rebrousse-poil de l'archive tend plutôt à repérer les dispositifs de pouvoir et les mécanismes de censure qui constituent et délimitent l'archive queer, ses pertes et ses résistances. Lire en suivant le grain enfin permet de faire place à l'altérité et aux contradictions d'un passé pervers subalterne, qui ne se laisse pas assimiler aux récits contemporains des démocraties libérales et nationalistes. Je défends ici l'idée que tordre les archives constitue une orientation éthique, cherchant à résister aux appels homo-nationalistes contemporains, ainsi qu'aux cloisonnements de l'histoire de la sexualité et de celle de l'empire colonial français.

The back and forth between epistemological questioning, political positioning, and affective experiences allows me to unpack several directions of the project of queering the archive. Michael Rosenfeld's edition of letters sent by an invert to Émile Zola and Dr. Saint-Paul at the end of the 19th century is, in this article, the occasion to identify a number of transformations in my relationship to the queer past, by distinguishing three ways of reading these letters that I have developed though my research. The project of a History of Sexual Minorities aims at discovering and rescuing the queer voices of the past, and seeks to do them justice in order to inspire the liberation struggles of the present. A reading of the archive against the grain tends instead to identify the devices of power and the mechanisms of censorship that constitute and delimit the queer archive, its losses and resistances. Reading along the grain finally makes room for the otherness and contradictions of a subaltern perverse past, which cannot be assimilated to the contemporary narratives of liberal and nationalist democracies. I defend here the idea that queering the archives constitutes an ethical orientation, seeking to resist contemporary homonationalist calls, as well as the separation of the history of sexuality from that of the French colonial empire. 
INDEX

Thèmes : Recherches

Keywords : archives, feelings, epistemology, queer, race

Mots-clés : archives, émotions, épistémologie, queer, race

\section{AUTEUR}

RUBY FAURE

Ruby Faure est doctorant·e en études de genre et philosophie, rattachéee au LEGS (Université Paris 8), sous la co-direction de Marie Dominique Garnier et de Guillaume Sibertin-Blanc, sur le thème : «Généalogie coloniale de l'homosexualité masculine européenne. Émergence des catégories sexo-raciales dans l'Empire français (1869-1912). » Contact : faure.ruby@gmail.com 